\title{
Radiation-induced ovarian follicle loss occurs without overt stromal changes
}

\author{
Bruce F Kimler ${ }^{1}$, Shawn M Briley², Brian W Johnson ${ }^{3}$, Austin G Armstrong ${ }^{2}$, Susmita Jasti ${ }^{2}$ and \\ Francesca E Duncan ${ }^{2,4}$ \\ ${ }^{1}$ Department of Radiation Oncology, University of Kansas Medical Center, Kansas City, Kansas, USA, ${ }^{2}$ Department \\ of Anatomy and Cell Biology, University of Kansas Medical Center, Kansas City, Kansas, USA, ${ }^{3}$ Department of \\ Comparative Medicine, University of Washington, Seattle, Washington, USA and ${ }^{4}$ Department of Obstetrics and \\ Gynecology, Feinberg School of Medicine, Northwestern University, Chicago, Illinois, USA
}

Correspondence should be addressed to F E Duncan; Email: f-duncan@northwestern.edu

\begin{abstract}
Radiation damage due to total body irradiation (TBI) or targeted abdominal radiation can deplete ovarian follicles and accelerate reproductive aging. We characterized a mouse model of low-dose TBI to investigate how radiation affects the follicular and stromal compartments of the ovary. A single TBI dose of either $0.1 \mathrm{~Gy}$ or $1 \mathrm{~Gy}$ (Cesium-137 $\gamma$ ) was delivered to reproductively adult CD1 female mice, and sham-treated mice served as controls. Mice were euthanized either 2 weeks or 5 weeks post exposure, and ovarian tissue was harvested. To assess the ovarian reserve, we classified and counted the number of morphologically normal follicles in ovarian histologic sections for all experimental cohorts using an objective method based on immunohistochemistry for an oocytespecific protein (MSY2), 0.1 Gy did not affect that total number of ovarian follicles, whereas $1 \mathrm{~Gy}$ resulted in a dramatic loss. At two weeks, there was a significant reduction in all preantral follicles, but early antral and antral follicles were still present. By five weeks, there was complete depletion of all follicle classes. We examined stromal quality using histologic stains to visualize ovarian architecture and fibrosis and by immunohistochemistry and quantitative microscopy to assess cell proliferation, cell death and vasculature. There were no differences in the ovarian stroma across cohorts with respect to these markers, indicating that this compartment is more radio-resistant relative to the germ cells. These findings have implications for reproductive health and the field of fertility preservation because the radiation doses we examined mimic scatter doses experienced in typical therapeutic regimens. Reproduction (2018) 155 553-562
\end{abstract}

\section{Introduction}

Radiation is a mainstay of treatment for most cancers, and total body irradiation (TBI) is used as a conditioning treatment for bone marrow transplantation. Despite the ability of radiation to target cancer cells, it can also have unintended off-target and long-term consequences on other organ systems. The extent of radiation-induced tissue damage is largely dependent on factors such as patient age, treatment dose and regimen, and radiation field (Meirow et al. 2010). Radiation can negatively impact all aspects of the female reproductive axis. For example, radiation directed to the cranium can damage the hypothalamus and pituitary leading to altered neuroendocrine function (Wo \& Viswanathan 2009). Radiation exposure to the uterus can decrease uterine weight and size, impair endometrial thickening and disrupt the vasculature and blood flow (Critchley $\&$ Wallace 2005). Such uterine alterations can increase the risk of miscarriage, placental abnormalities, preterm birth and delivery of low-birth-weight offspring (Wo \& Viswanathan 2009).
The ovary is particularly susceptible to radiation damage, with the dose required to eliminate half the primordial follicles in the ovary estimated at $2 \mathrm{~Gy}$ (Wallace et al. 2003). The follicle is the functional unit of the ovary and consists of a germ cell surrounded by supporting somatic cells. Follicles produce endocrine hormones and are essential for generating mature gametes capable of being fertilized. Thus, the number of follicles within the ovary is an important readout of reproductive function. Females are born with a finite number of primordial follicles, which dictate the ovarian reserve and reproductive lifespan. During physiologic reproductive aging, the number of follicles within the ovary steadily declines through processes of recruitment and cell death, such that females are born with approximately 1 million primordial follicles but end up with only about 1000 at the time of menopause (Broekmans et al. 2009). Factors such as radiation accelerate reproductive aging. Importantly, there is no evidence of follicular renewal following radiationinduced depletion, and thus, exposure can result in 
premature ovarian failure, subfertility and infertility (Kerr et al. 2012a). In fact, ovarian failure has been reported in $90 \%$ of females who received TBI and in $97 \%$ of those who received abdominal radiation (Wallace et al. 1989, Sanders et al. 1996). Therefore, understanding the cellular and molecular mechanisms that underlie radiation-induced ovarian damage has important implications for reproductive health.

To date, a significant amount of research has pinpointed DNA damage checkpoints and intrinsic apoptosis pathways as critical regulators of radiation-induced cell death within the oocyte (Kerr et al. 2012b, Hutt 2015, Rinaldi et al. 2017). In fact, genetic and pharmacologic manipulations of these pathways have been harnessed for fertoprotection efforts to preserve germ cells and endocrine function in response to radiation damage (Morita et al. 2000, Zelinski et al. 2011, Rinaldi et al. 2017). However, less is known about the effect of radiation on the ovarian microenvironment especially in response to low doses of radiation that are known to affect the follicle. The ovarian microenvironment consists of the stroma or a mixture of extracellular matrix components as well as theca-interstitial, immune, endothelial and smooth muscle cells. The ovarian stroma provides the physical and signaling milieu in which follicles grow and develop. Thus, damage to the stroma can have important long-term ramifications on germ cell health and reproductive function. In this study, we characterized the simultaneous effect of low-dose radiation exposure on both the follicular and stromal components of the ovary in a reproductively mature animal model of TBI.

\section{Materials and methods}

\section{Animals, irradiation paradigm and ovarian tissue harvesting}

CD1 female mice were obtained from Envigo (Indianapolis, IN, USA) and were housed in a controlled barrier facility at the University of Kansas Medical Center's (KUMC) Research Support Facility under constant temperature, humidity and light (12-h light/12-h dark). Food and water were provided ad libitum. All experimental protocols were approved by the Institutional Animal Care and Use Committee of the University of Kansas Medical Center and were in accordance with National Institutes of Health Guidelines.

At 6 weeks of age, mice were exposed to a single dose of 0.1 Gy or 1 Gy TBI using a Model MK I-68 Cesium-137 Irradiator (J L Shepherd and Associates, San Fernando, CA, USA) (Supplementary Fig. 1, see section on supplementary data given at the end of this article). A 302 shaped attenuator was used to produce a uniform field and a dose rate of $2.1 \mathrm{~Gy} /$ min. A $10 \times$ attenuator was added for the low-dose irradiation. With these conditions, all irradiations took $48 \mathrm{~s}$ per mouse. During this time, the mice were confined to standardize exposure. Sham-treated mice served as controls. These mice were handled in the same manner as the experimental cohorts but were not exposed to radiation. Following irradiation, mice were group housed in sterile cages.

Animals in each cohort (0.1 Gy, 1 Gy, Sham) were killed either 2 or 5 weeks post irradiation and ovarian tissue was harvested (Supplementary Fig. 1). Ovaries were fixed in Modified Davidson's (Electron Microcopy Sciences, Hatfield, $\mathrm{PA}, \mathrm{USA}$ ) for $6 \mathrm{~h}$ at room temperature and then overnight at $4^{\circ} \mathrm{C}$. After fixation, these ovaries were dehydrated using an automated tissue processor (Leica Biosystems), embedded in paraffin and serially sectioned ( $5 \mu \mathrm{m}$ thickness). For all experiments described below, ovarian tissue from a minimum of 3 animals per cohort was examined and representative images were analyzed and are shown.

\section{Follicle identification and counting}

Follicles were counted in every 5th section throughout each ovary according to previously published protocols (BristolGould et al. 2006, Duncan et al. 2017). Follicle stages were classified according to morphological criteria, and only healthy follicles were counted (Duncan et al. 2017). Primordial follicles were classified as an oocyte surrounded by squamous granulosa cells, whereas primary follicles were characterized as oocytes surrounded by a complete single layer of cuboidal granulosa cells. All primordial and primary follicles were counted irrespective of whether the oocyte nucleus was visible in the section. Secondary follicles contained a larger oocyte surrounded by more than one layer of granulosa cells. Early antral follicles contained a developing antral cavity, and antral follicles were the largest follicles with prominent antral spaces. For secondary through antral stages, only follicles with an oocyte nucleus present in the section were counted to avoid double counting. The total follicle count for each stage per ovary was then divided by the total number of sections counted per ovary to obtain an average number of follicles per section.

The objectivity of the follicle counting procedure was enhanced by performing immunohistochemistry on sections used for follicle counting with an antibody against the oocyte-specific marker MSY2 (generous gift of Dr Richard M. Schultz, University of Pennsylvania). Only follicles containing MSY2-positive oocytes were counted. In brief, slides were deparaffinized in CitriSolv (Thermo Fisher) and rehydrated in a series of graded ethanol baths $(100,95,85,70$ and 50\%). Antigen retrieval was performed by microwaving slides in $1 \mathrm{X}$ Reveal Decloaker (BioCare Medical, Pacheco, CA, USA) at $50 \%$ power for two minutes followed by $10 \%$ power for seven minutes. Slides were washed with Tris-buffered saline supplemented with $0.1 \%$ Tween-20 (TBST) twice for $15 \mathrm{~min}$ and then incubated in 3\% hydrogen peroxide for $15 \mathrm{~min}$ at room temperature. Slides were briefly rinsed in Tris-buffered saline (TBS) and blocked using the avidin/biotin blocking kit (Vector Laboratories, Burlingame, CA, USA). The slides were briefly rinsed with TBS and the area around the tissue was defined using a PAP pen. The slides were incubated in blocking buffer (10\% normal goat serum, 0.3\% Triton X-100 in TBS) for one hour. The blocking buffer was removed and the slides were incubated in primary antibody diluted in blocking buffer $(1: 4000)$ overnight at $4^{\circ} \mathrm{C}$. Slides were then rinsed three 
times in TBST for 5 min each. Secondary antibody incubation and horseradish peroxidase (HRP) conjugation was performed using the VECTASTAIN Elite ABC HRP Kit according to the provided instructions. Detection was performed using 3,3'-diaminobenzidine (DAB) using the DAB Peroxidase (HRP) Substrate Kit according to the manufacturer's instructions (Vector Laboratories). The reaction was allowed to proceed for $1 \mathrm{~min}$. Counterstaining was performed as follows: 1-min incubation in hematoxylin, $2 \mathrm{~min}$ in running water, $20 \mathrm{~s}$ in acid ethanol, running water for $1 \mathrm{~min}$, hematoxylin for $1 \mathrm{~min}$, a brief rinse in water, bluing reagent for $1 \mathrm{~min}$ and running water for one minute. The slides were then dehydrated in graded ethanol baths (80, 95 and 100\%), cleared in CitriSolv and mounted with Cytoseal.

\section{Histologic staining}

Ovarian histologic sections from each animal in all experimental cohorts were stained with hematoxylin and eosin (H\&E), Periodic Acid Schiff (PAS) and Picrosirius Red (PSR) as previously described (Briley et al. 2016). To view entire ovary sections, individual $40 \times$ brightfield images were taken and stitched together using an EVOS FL Auto Cell Imaging system (Thermo Fisher).

\section{Automated immunohistochemistry and quantitative microscopy}

We performed automated immunohistochemistry through the University of Washington Histology and Imaging Core (UW-HIC) with antibodies specific to Ki67 (Clone D3B5, Cell Signaling), cleaved caspase 3 (CC3, BioCare Medical) and CD31 (Clone SZ31, Dianova, Hamburg, Germany) to assess proliferation, apoptosis and vasculature, respectively, in ovarian histologic sections from all experimental cohorts. In brief, slides were baked for $30 \mathrm{~min}$ at $60^{\circ} \mathrm{C}$ and deparaffinized on the Leica Bond MAX Automated Immunostainer (Leica Microsystems) using Leica Bond Dewax Solution (Leica Cat No. AR922). Antigen retrieval was performed on all slides using EDTA solution pH 9.0 (Lieca Bond Epitope Retrieval Solution 2, Cat No AR9640) at $100^{\circ} \mathrm{C}$ for 10 or 20 min depending on the antibody used. Blocking consisted of $10 \%$ normal goat serum in TBS buffer for $20 \mathrm{~min}$ at room temperature. The primary antibodies were diluted as follows in Leica Bond Primary Antibody Diluent (Leica Cat No. AR9352): Ki67 (1:400), CC3 (1:100), and CD31 (1:50). Slides were incubated with Ki67 and CD31 primary antibodies for $30 \mathrm{~min}$ at room temperature. Remaining slides were incubated with CC3 antibody for $60 \mathrm{~min}$ at room temperature. Slides probed with CD31 primary antibody were then incubated with unconjugated rabbit antirat $\operatorname{lgG}(\mathrm{H}+\mathrm{L})$, mouse adsorbed, secondary antibody for $8 \mathrm{~min}$ at room temperature. All slides were incubated with goat anti-rabbit poly-HRP polymerized secondary detection (Leica Cat No DS9800) for $8 \mathrm{~min}$ at room temperature. Additional blocking for endogenous peroxidase was then performed using Leica peroxide block $\left(3 \% \mathrm{H}_{2} \mathrm{O}_{2}\right.$ /Leica Cat No DS9800) for $5 \mathrm{~min}$ at room temperature. Sections were then incubated with Leica Bond Mixed Refine DAB substrate detection for 10 min at room temperature. (Leica Cat No DS9800). Antibody complexes were visualized using Leica Bond Mixed Refine (DAB, 3, 3'-diaminobenzidine) detection $2 \times$ for $10 \mathrm{~min}$ at RT (Leica Cat No DS9800). Tissues were counterstained with hematoxylin counterstain for 4 min followed by two rinses in water. Slides were then dehydrated through $100 \% \mathrm{ETOH}$, cleared in xylene and mounted with synthetic resin-mounting medium and a \#1.5 coverslip.

Slides were scanned in brightfield with a $20 \times$ objective using the Hamamatsu NanoZoomer Digital Pathology System HT9600 (Hamamatsu City, Japan). The digital images were then imported into Visiopharm software (Hoersholm, Denmark) for analysis. Using the Visiopharm Image Analysis module, three regions of interests (ROI) were manually drawn around the follicles, corpora lutea and the stroma respectively. In addition, the entire area of the ovary was determined. Follicles were identified as the functional units of the ovary containing an oocyte surrounded by layers of granulosa cells all contained within a visible basement membrane. Corpora lutea were identified based on the morphology of luteinized cells, which have a characteristic hypertrophied and eosinophilic appearance relative to non-luteinized granulosa and theca cells (Supplementary Fig. 4D). Of note, we only classified structures as corpora lutea if there was a clear boundary enclosing the luteinized cells. Thus, it is likely that degrading corpora lutea from previous cycles were considered as interstitial stromal cells. The stromal area was defined as the total ovarian area minus the sum of the total follicular area and the total corpora lutea area. By converting the initial digital image into grayscale values using two features, $\mathrm{HDAB}-\mathrm{HDAB}$ and $\mathrm{RGB}-\mathrm{B}$, the Visiopharm software was trained to label positive staining (DAB) and background tissue counterstain (hematoxylin) using a project-specific configuration based on a threshold of pixel values. The images were processed in batch mode using this configuration to generate the desired per area outputs and analyzed at 100\%. Ovarian histologic sections from each animal in all experimental cohorts were analyzed.

\section{Statistical analysis}

Significant changes between groups were analyzed by oneway ANOVA or two-way ANOVA followed by multiple comparisons test. $P$ values $<0.05$ were considered statistically significant. Statistical analysis was performed using Graphpad Prism Software, version 6.0f.

\section{Results}

\section{TBI depletes ovarian follicles in a dose- dependent manner}

To examine how ovarian follicles respond to low doses of radiation, we established an adult animal model of TBI (Supplementary Fig. 1). Reproductively adult CD1 female mice were exposed to a single dose of either $0.1 \mathrm{~Gy}$ or $1 \mathrm{~Gy}$ of $\gamma$ radiation delivered uniformly to the whole body. Ovaries were then harvested either 2 or 5 weeks post exposure, and follicles were classified by stage according to morphology and quantified. Typically, follicles are evaluated in H\&E- stained histologic 
sections, but this method is subjective especially when trying to identify small primordial follicles in the ovarian cortex (Supplementary Fig. 2A and B). To improve the objectivity of follicle counting, we immunostained ovarian histologic sections using an antibody against MSY2, an abundant oocyte-specific protein that localizes to the cytoplasm (Supplementary Fig. 2C and D) (Yu et al. 2002). This marker clearly delineated oocytes and improved the ability to identify and quantify follicles relative to sections that were stained with $\mathrm{H} \& \mathrm{E}$ alone (Supplementary Fig. 2).

Using this follicle detection strategy, we determined that a single-dose exposure to $0.1 \mathrm{~Gy}$ radiation did not cause a significant decrease in the number of follicles. At 2 weeks post irradiation, average follicle counts were $33.2 \pm 2.2$ follicles/section in irradiated mice compared to $43.6 \pm 8.5$ follicles/section in sham-treated age-matched mice, but this change was not significant (Fig. 1A, B and G). At 5 weeks post irradiation, the average number of follicles per section was $22.4 \pm 1.2$ in irradiated mice compared to $33.1 \pm 3.0$ in the controls, and again, this difference was not significant (Fig. 1D, $\mathrm{E}$ and $\mathrm{G}$ ). In contrast to $0.1 \mathrm{~Gy}$ radiation, $1 \mathrm{~Gy}$ radiation caused a significant depletion of follicles compared to controls at both the 2 and 5 week time points (Fig. 1C, F and $\mathrm{G})$. Post exposure to $1 \mathrm{~Gy}$ radiation, there were only $1.6 \pm 0.1$ follicles/section at 2 weeks, and no follicles remaining at 5 weeks (Fig. 1G). This average follicle number was also significantly less than that observed in the ovaries from 0.1 Gy-exposed mice at both time points (Fig. 1G). These findings demonstrate that in adult CD1 mice, exposure to $\gamma$ radiation causes a dosedependent reduction in the total number of ovarian follicles with 1 Gy causing significantly more damage compared to $0.1 \mathrm{~Gy}$.

\section{A single-dose exposure to 1 Gy TBI differentially affects follicle classes}

Although 1 Gy exposure caused a significant reduction in total follicle numbers relative to ovaries from mice that were exposed to $0.1 \mathrm{~Gy}$ radiation or sham controls, histologic evaluation demonstrated that the follicle classes affected were different between the 2 and 5 week time points (Figs 2 and 3). Ovaries from mice that received a single dose of $1 \mathrm{~Gy}$ radiation lacked primordial follicles in the ovarian cortex at both 2 and 5 weeks post exposure, and this was in contrast to mice exposed to 0.1 Gy radiation and sham controls, which all showed evidence of primordial follicles (Fig. 2). However, although no follicles were present in ovaries from 1 Gy-exposed mice following 5 weeks post treatment, large growing follicles were still visible at 2 weeks (Fig. 2C and F).

To further quantify these phenotypes, we obtained follicle counts across different classes and normalized
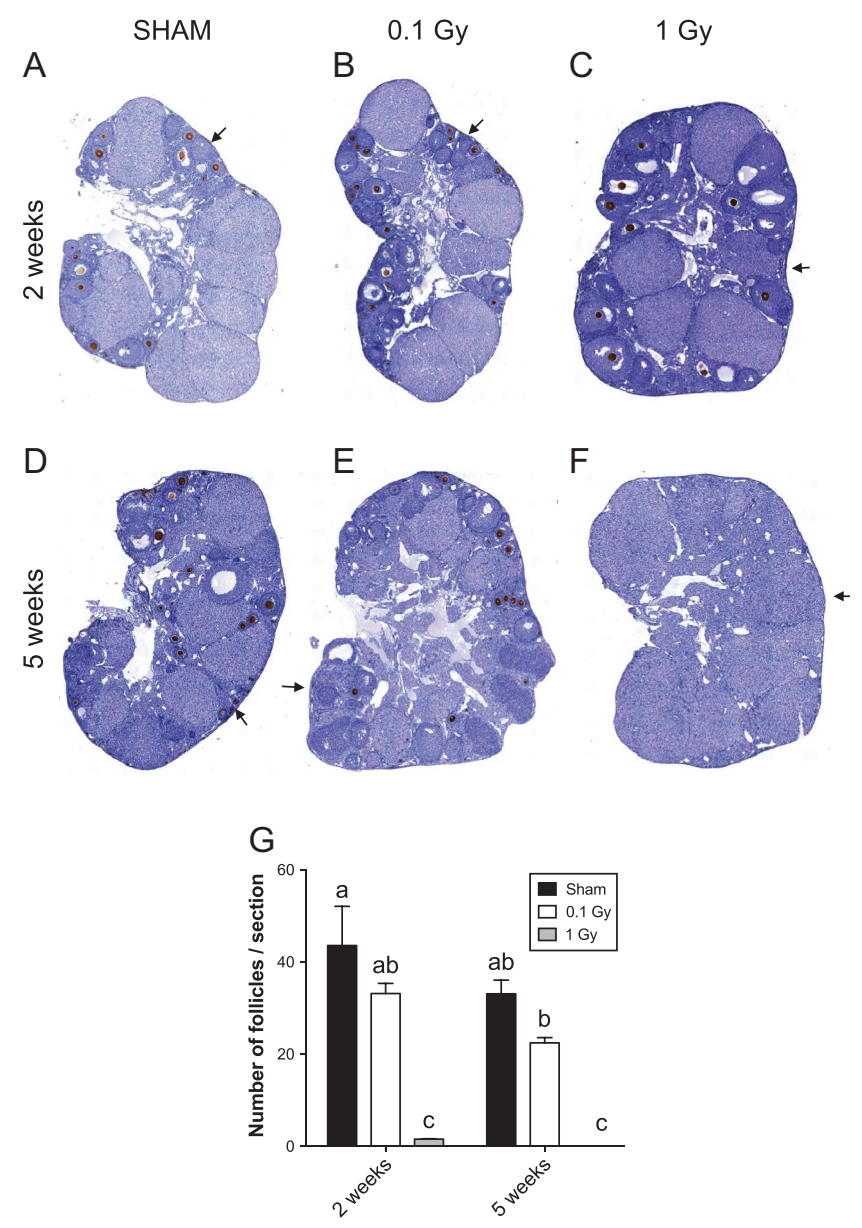

Figure $1 \mathrm{~A}$ single dose of $1 \mathrm{~Gy}$ TBI depletes total follicle numbers by 2 weeks post exposure. Histologic sections of ovarian tissue from (A and D) control sham mice and mice exposed to a single dose of ( $B$ and E) 0.1 Gy or (C and F) 1 Gy that were harvested either (A, B and C) 2 weeks or (D, E and F) 5 weeks post exposure were stained with the oocyte-specific MSY2 antibody (brown, DAB) and counterstained with hematoxylin. Representative images are shown. (G) Follicle numbers in all follicle classes were quantified for each cohort and reported as the average number of follicles per section per ovary. The different letters denote a statistically significant difference between groups as assessed by a two-way ANOVA $(P \leq 0.01)$. The regions highlighted by the arrow are further magnified in Fig. 2.

counts to the sham control (Fig. 3). At 2 weeks post exposure, $1 \mathrm{~Gy}$ radiation completely eliminated both primordial and primary follicles and significantly reduced the number of secondary follicles relative to mice exposed to $0.1 \mathrm{~Gy}$ radiation or sham controls (Fig. 3A). There were no differences in the number of early antral follicles between the experimental cohorts, and there was even an increase in the number of antral follicles in mice exposed to $1 \mathrm{~Gy}$ radiation relative to both $0.1 \mathrm{~Gy}$ and the sham control (Fig. 3A). In contrast and consistent with the histology, 1 Gy radiation completely eradicated all follicle classes at 5 weeks post 

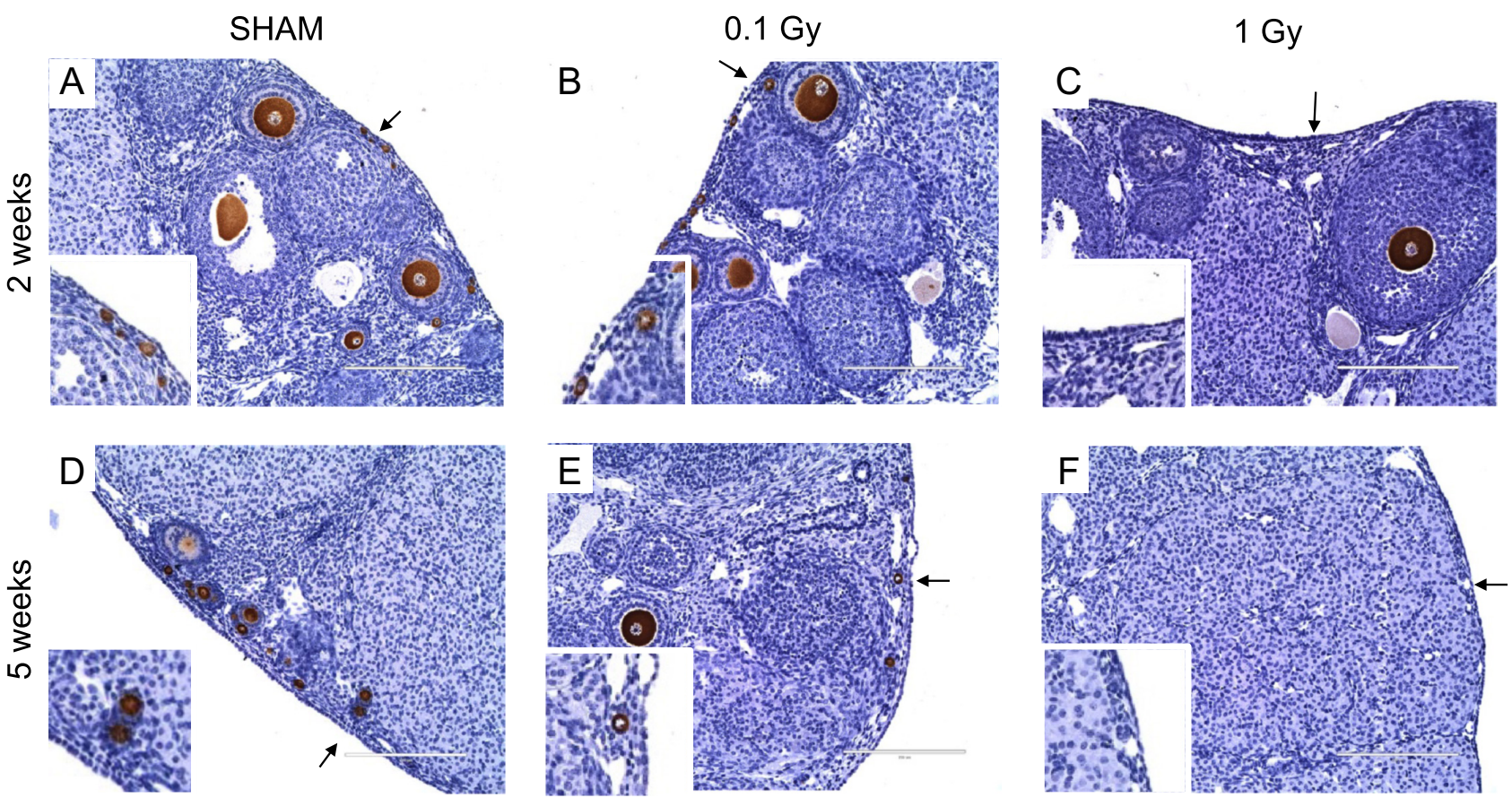

Figure 2 Histologic analysis demonstrates that primordial and primary follicles are absent in the ovarian cortex following exposure to a single dose of 1 Gy radiation. (A, B, C, D, E and F) show magnified images of the ovary in the corresponding regions highlighted in Fig. 1. The insets show specific regions of the ovarian cortex that are marked by the arrows. These images illustrate the significant follicle damage that occurs in response to a single dose of $1 \mathrm{~Gy}$ radiation at both 2 weeks and 5 weeks post exposure. Note the clear lack of primordial and primary follicles in both (C) and (F). In addition, large growing follicles are only observed at 2 weeks post exposure to $1 \mathrm{~Gy}(\mathrm{C})$ and not at 5 weeks (F). Scale bars are $200 \mu \mathrm{m}$.

exposure relative to both $0.1 \mathrm{~Gy}$ and the sham control, and there were no differences across the follicle classes in the 0.1 Gy and sham cohorts (Fig. 3B). Taken together, these results demonstrate that 1 Gy radiation rapidly eliminates the majority of dormant and early growing follicles in the ovary, but that a subset of follicles can survive and grow to antral stages following shortterm exposure.
A

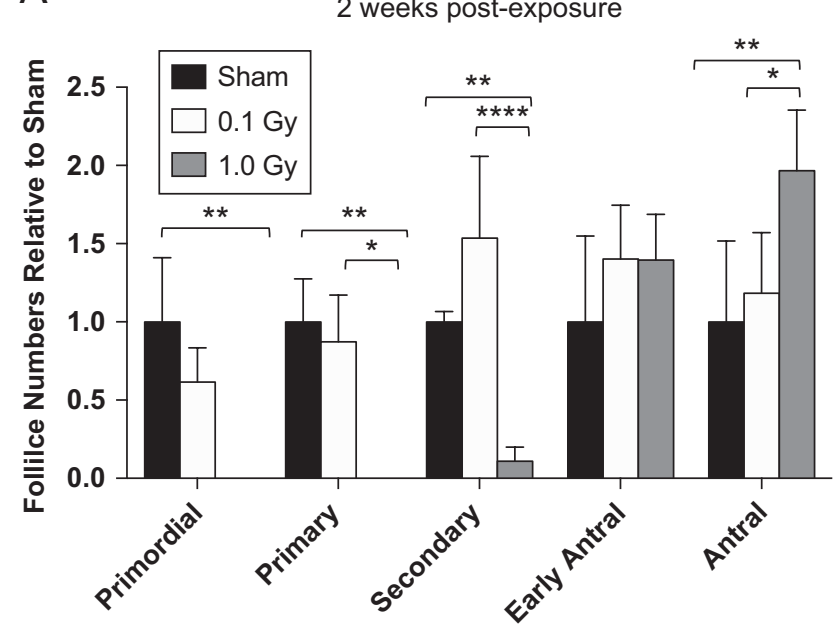

B

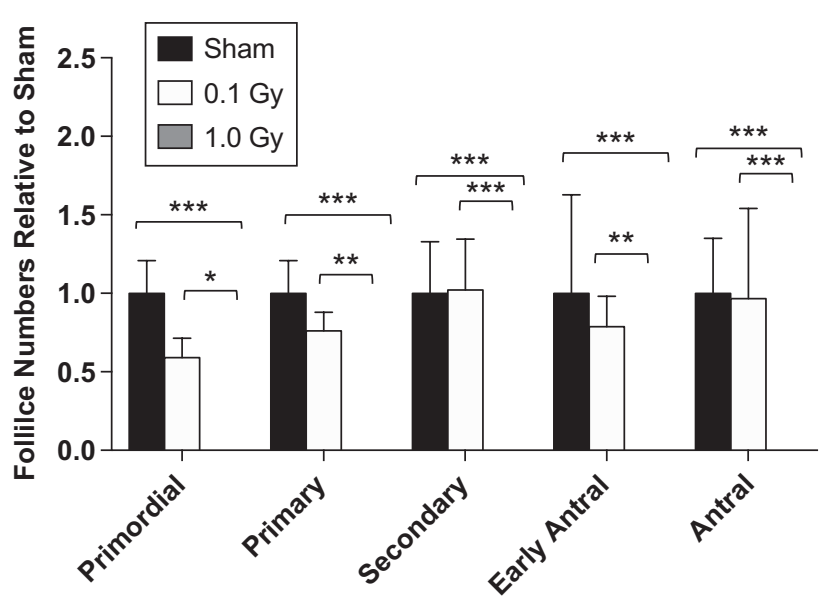

Figure 3 Stage-dependent differences in ovarian follicle numbers occur in response to single doses of 0.1 and 1 Gy TBI. Follicle stages were classified and counted for each experimental cohort (sham, $0.1 \mathrm{~Gy}$ and $1 \mathrm{~Gy}$ ), and the average follicle numbers per section in each class were expressed relative to the average number of follicles in the sham controls at (A) two weeks and (B) five weeks post exposure. Statistically significant differences between the groups were assessed by a two-way ANOVA, and asterisks highlight significant differences $(* P \leq 0.05$, $\left.{ }^{* * P} \leq 0.01,{ }^{* * *} P \leq 0.001,{ }^{* * * *} P \leq 0.0001\right)$. Of note, in the 1 Gy cohort (gray bars), only growing follicles remained at 2 weeks but no follicles were observed at 5 weeks. 


\section{The ovarian stroma is resistant to doses of TBI that are sufficient to deplete ovarian follicles}

To investigate how the ovarian stroma was affected by the same dose of radiation that significantly damaged the ovarian reserve, we performed a histologic analysis of ovaries from mice that were treated with 1 Gy radiation and harvested 2 and 5 weeks post exposure (Fig. 4). We stained ovarian tissue sections with $\mathrm{H} \& \mathrm{E}$ to evaluate general tissue morphology (Fig. 4A, B, C and D). We did not observe obvious gross morphological differences across cohorts except for the complete absence of follicles in ovaries from mice that were harvested at 5 weeks post exposure (Fig. 4D). Interestingly, the remaining tissue in these ovarian sections had a luteinized appearance characterized by eosinophilic and hypertrophied cells analogous to the corpora lutea in the other experimental cohorts (Fig. 4A, B, C and D). We also stained sections with PAS, which detects polysaccharides. We previously used PAS to visualize multinucleated macrophage giant cells in fibrotic ovarian tissue from mice of advanced reproductive age (Briley et al. 2016). However, here we did not observe any differences in PAS staining across experimental cohorts (Fig. 4E, F, G and H). Finally, we stained sections with PSR, a histologic stain specific for collagen I and III, which is often used to detect fibrosis (Briley et al. 2016). We did not observe prominent differences in PSR staining among ovaries from the different experimental cohorts suggesting that radiation exposure did not induce ovarian fibrosis. Not surprisingly, we also did not observe differences in H\&E, PAS and PSR staining in ovaries from mice exposed to $0.1 \mathrm{~Gy}$ radiation relative
SHAM, 2 weeks

A

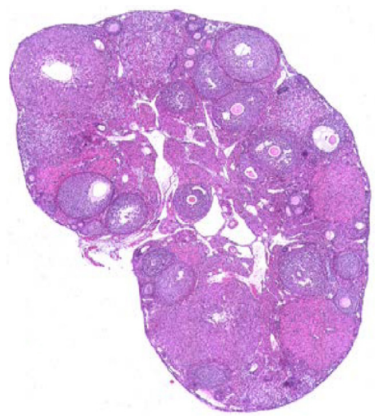

E

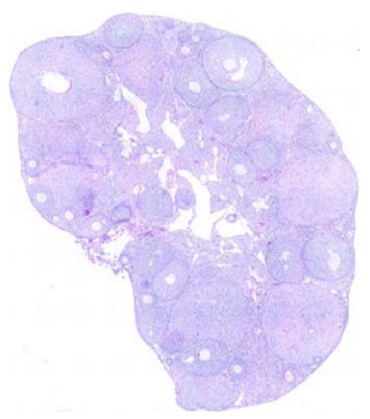

I

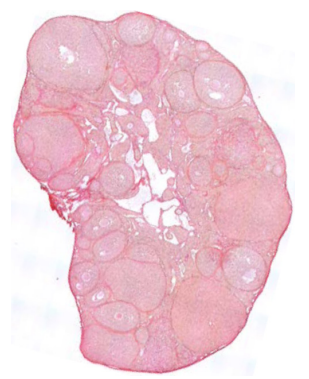

$1 \mathrm{~Gy}, 2$ weeks

B

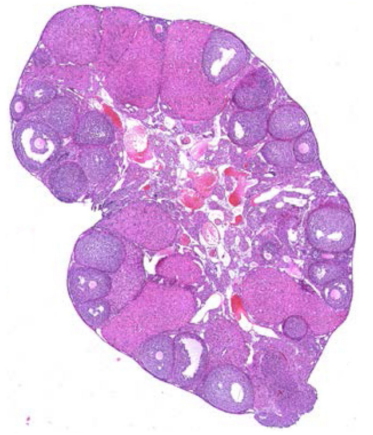

F

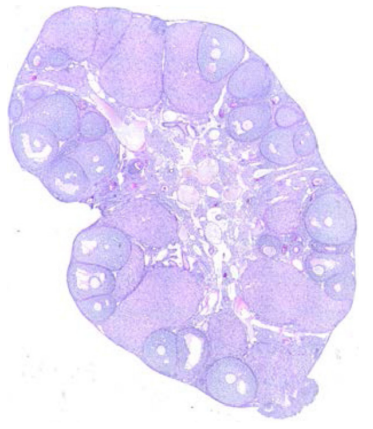

J

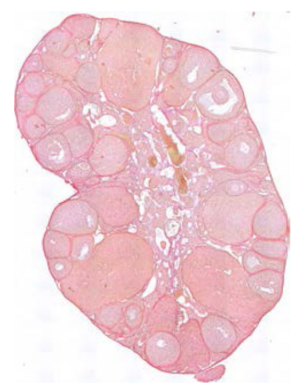

SHAM, 5 weeks

C

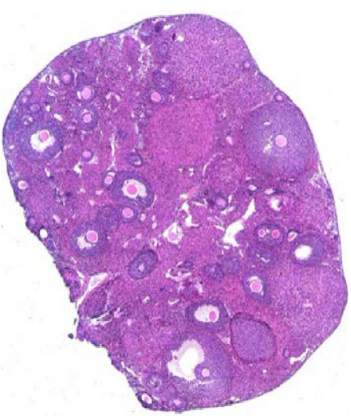

G

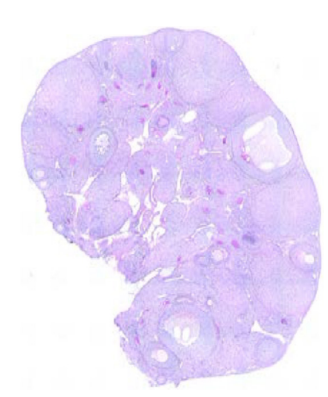

K

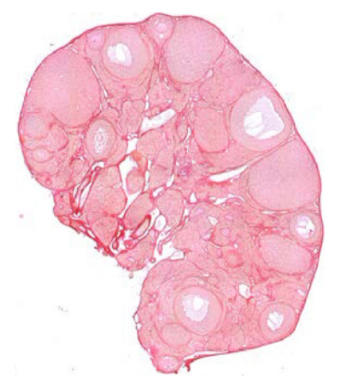

$1 \mathrm{~Gy}, 5$ weeks

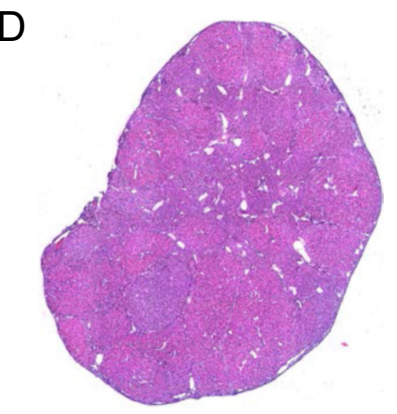

$\mathrm{H}$

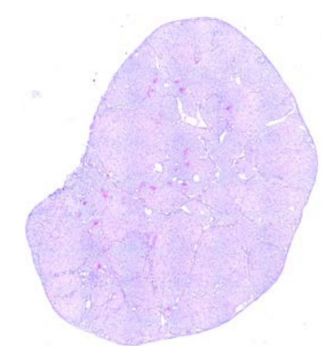

L

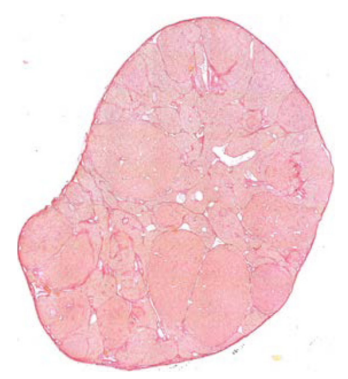

Figure 4 Histologic stains reveal healthy ovarian stromal tissue following a single-dose exposure to 1 Gy TBI at 2 and 5 weeks post exposure. Ovaries from (A, C, E, G, I and K) sham mice or (B, D, F, H, J and L) mice exposed to 1 Gy radiation were harvested either two weeks (left two image columns) or five weeks (right two image columns) post exposure and histologic sections were stained for (A, B, C and D) general architecture (hematoxylin and eosin), (E, F, G and H) polysaccharides (Periodic Acid Schiff), and (I, J, K and L) collagen I/III (Picrosirius Red). Representative images are shown. 
to sham controls at either the 2 week or 5 week time points (Supplementary Fig. 3).

To further examine how low-dose exposure to radiation affects the ovarian stroma, we optimized and validated automated immunohistochemistry protocols on ovarian tissue sections for two markers of general tissue health, including Ki67 for proliferating cells and cleaved caspase 3 (CC3) for apoptotic cells (Supplementary Fig. 4A and B). Ki67 refers to the antigen recognized by a monoclonal antibody that was generated by immunizing mice with nuclei of a Hodgkin lymphoma cell line (Scholzen \& Gerdes 2000). Although the exact function of this protein remains elusive, it is expressed in nuclei of cells in G1, S, G2 and M phases but not in resting cells and, therefore, is commonly used as a proliferation marker (Scholzen \& Gerdes 2000). On the other hand, CC3 is an effector caspase involved in the initiation of the cell death pathway and is often used as a marker of cellular apoptosis (Nicholson et al. 1995). Using quantitative immunohistochemistry approaches, we analyzed Ki67 and CC3 staining in ovarian tissue sections across experimental cohorts in specific compartments of the ovarian tissue (follicles, corpora lutea, and stroma) and quantified the number of cells positive for each marker (Fig. 5A, B and C). In general, more proliferative (Ki67 positive) and apoptotic cells (CC3 positive) were observed in follicles relative to the corpora lutea and stroma (Fig. 5D, E, F, G, H and I). There was an increase in the number of proliferating cells in follicles from the sham cohort between the 2-week and 5-week time point (Fig. 5A). In addition, at the 2-week time point, there was an increase in the number of proliferating cells in follicles following exposure to $1 \mathrm{~Gy}$ radiation relative to the sham controls, consistent with the preservation and increase in early antral and antral follicles, respectively, in the 1 Gy cohort (Figs 3A and 5A). Proliferating follicle cells were obviously not observed at 5 weeks in the 1 Gy cohort due to the complete depletion of follicles (Figs 1G, 3B, and 5D). No differences in proliferation were observed across experimental cohorts in the corpora lutea or the stroma (Fig. 5E and F). Moreover, the number of apoptotic cells did not change irrespective of treatment in any of the ovarian compartments (Fig. 5G, $\mathrm{H}$ and $\mathrm{I})$.

We also examined the architecture of the ovarian vasculature by performing immunohistochemistry with an antibody against CD31, a membrane glycoprotein that is expressed at high levels in early and mature endothelial cells (Fig. 6). Based on our histologic analysis, the CD31 staining pattern in the ovary was similar across the experimental cohorts and localized as expected. For example, in groups where growing follicles were still present in the tissue, the vasculature was prominent in the theca layer surrounding the follicles. In all groups, corpora lutea were visible in the histologic sections and were highly vascularized. The stromal and interstitial vessels were also similar across cohorts.

\section{Discussion}

In a sexually mature mouse model of $\mathrm{TBI}$, we report a clear dose response of ovarian follicles to ionizing radiation. To increase the objectivity and accuracy of follicle counting, we quantified follicle numbers in ovarian histologic sections by performing immunohistochemistry for an oocyte-specific marker. Using this method, we demonstrated that 1.0 Gy radiation caused a significant reduction in total follicle numbers at both 2 weeks and 5 weeks post exposure, whereas there was no such significant reduction in response to $0.1 \mathrm{~Gy}$ radiation at either time point. These results are in contrast to another study that showed a near-complete destruction of primordial follicles at 2 weeks following a single-dose exposure of 0.1 Gy TBI (Morita et al. 2000). Such inconsistencies underscore the need to consider the numerous variables, which can impact the effects of ionizing radiation when interpreting results, including animal age and strain, oocyte and follicle stage, the experimental design (e.g. radiation dose, rate, exposure and frequency) and the measured endpoints (Baker 1978). While we used an analogous experimental paradigm as the previous study, they did not report the mouse strain, which may account for the observed differences. For example, we used an outbred mouse strain, which may be more radioresistant compared to inbred strains due to their genetic heterogeneity.

In addition to a dose response, we also observed a follicle stage-dependent response to radiation. Early growing follicles from the primordial to secondary stages were significantly depleted in response to $1.0 \mathrm{~Gy}$ radiation, whereas later stage follicles were still present in either equal or greater numbers relative to sham controls. These findings are consistent with previous mouse and rat data demonstrating that early follicle stages have a high sensitivity to radiation and larger follicles have a low sensitivity (Adriaens et al. 2009). These persistent larger follicles likely derived from early growing follicles that escaped radiation damage.

In contrast to the changes we observed in ovarian follicles with the experimental paradigm of radiation exposure we used, the surrounding stroma remained unaffected at both doses of radiation based on gross markers of tissue health. In general, quantitative levels of stromal cell proliferation and death were indistinguishable between irradiated and sham cohorts at both 2 and 5 weeks post radiation exposure. In human ovaries, radiation exposure not only causes follicle loss, but it is also associated with documented changes in the ovarian stroma including increased cortical fibrosis (Grigsby et al. 1995). In addition, ionizing radiation can cause vascular damage in many tissues through induction of endothelial cell injury, and in ovaries from women who were treated with a sterilizing dose of radiation, there is an acceleration of spontaneous sclerosis of the ovarian arteries and arterioles (Grigsby et al. 1995). 
A

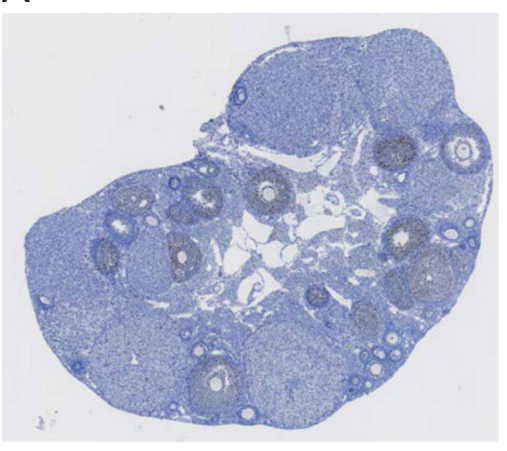

D

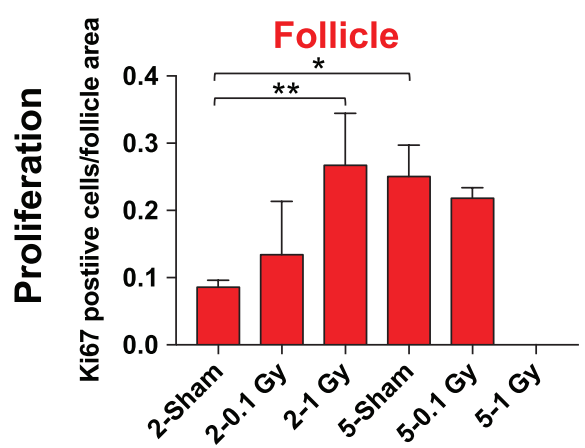

G

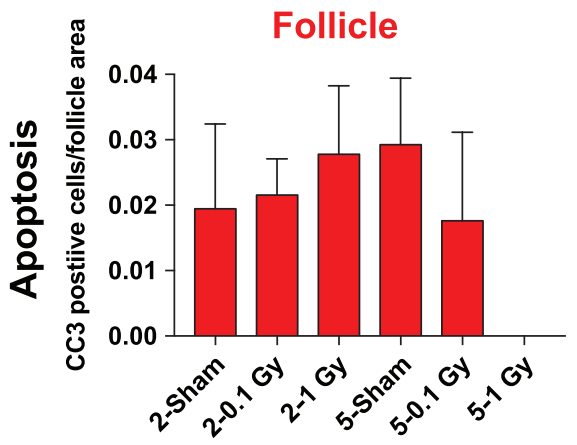

B

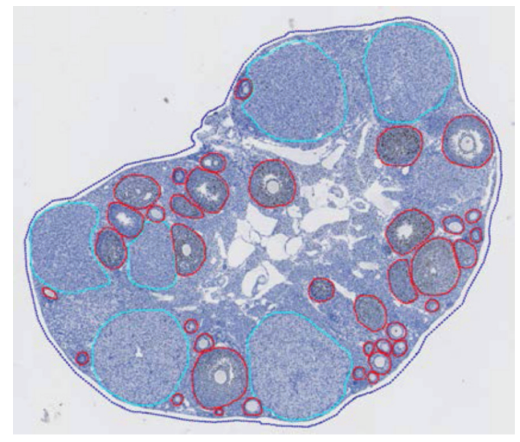

E

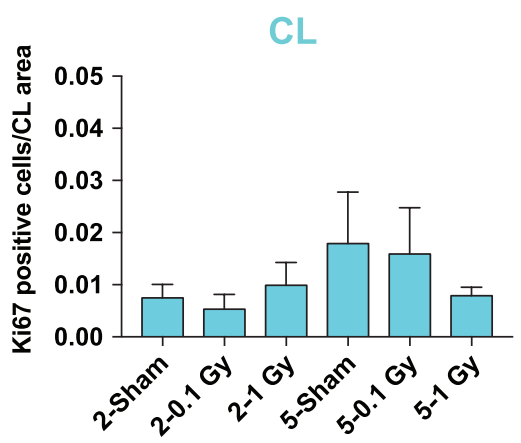

$\mathrm{H}$

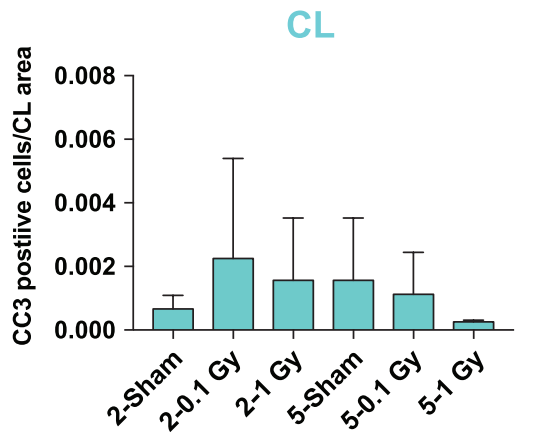

C

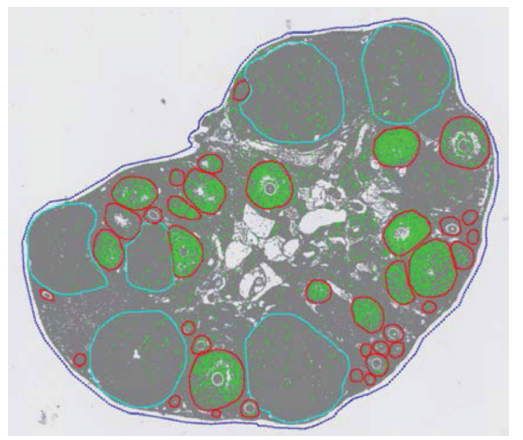

F

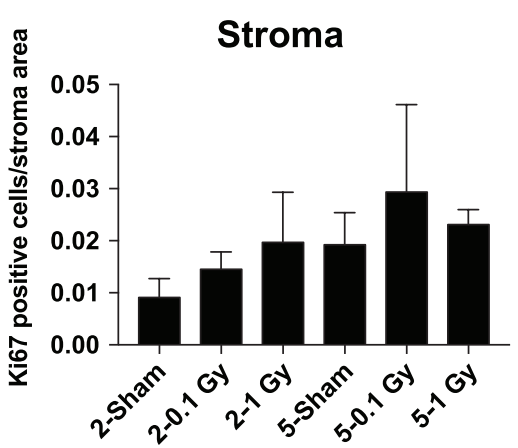

I

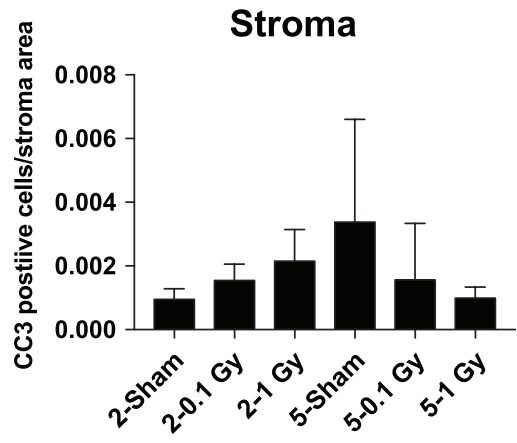

Figure 5 The ovarian stroma does not exhibit changes in cell proliferation or cell death in response to single doses of 0.1 and 1 Gy TBI at 2 and 5 weeks post-exposure. (A) Ovarian tissue sections were stained with an antibody against the cell proliferation marker, Ki67, using automated immunohistochemistry and whole slides were imaged. Quantitative microscopy was performed using Visiopharm software where (B) regions of interest (follicle - red, corpora lutea; $\mathrm{CL}$ - cyan, ovarian area - blue) were manually defined. (C) The Visiopharm software was trained to label and count positive (brown DAB) and background tissue counterstain (hematoxylin, blue) using a project-specific configuration based on a threshold of pixel values (green). The same analyses shown in (A, B and C) were done for histologic sections stained with the apoptosis marker CC3. Validation of these antibodies is shown in Supplementary Fig. 4. Using this approach, we were able to quantify the number of (D, E and F) proliferating or $(\mathrm{G}, \mathrm{H}$ and $\mathrm{I})$ apoptotic cells per specific ovarian area, including (D and $\mathrm{G}$ ) the follicle, (E and $\mathrm{H})$ the $\mathrm{CL}$ and $(\mathrm{F}$ and $\mathrm{I})$ the stroma. The stromal area was defined as the (total ovarian area - (follicle area $+\mathrm{CL}$ area)). Statistically significant differences between the groups were assessed by a one-way ANOVA, and asterisks highlight significant differences $\left({ }^{*} P \leq 0.05,{ }^{*} P \leq 0.01\right)$. Follicles were completely absent in the 1 Gy cohort at 5 weeks; hence, there was no proliferation or apoptosis noted in this ovarian compartment.

In our study, we did not detect prominent fibrosis or obvious changes in the ovarian vasculature at any of the radiation doses or time points investigated. This could be because single-dose exposures of either 0.1 Gy or $1 \mathrm{~Gy}$ ionizing radiation are not sufficient to induce these cellular responses in reproductively adult mice. In addition, it is possible that 5 weeks post irradiation may be too early a time point to observe chronic effects of radiation exposure. In fact, in humans, both radiationinduced vascular injury and tissue fibrosis are both long-term consequences of exposure, which can take up to years to manifest clinically (Weintraub et al. 2010, 

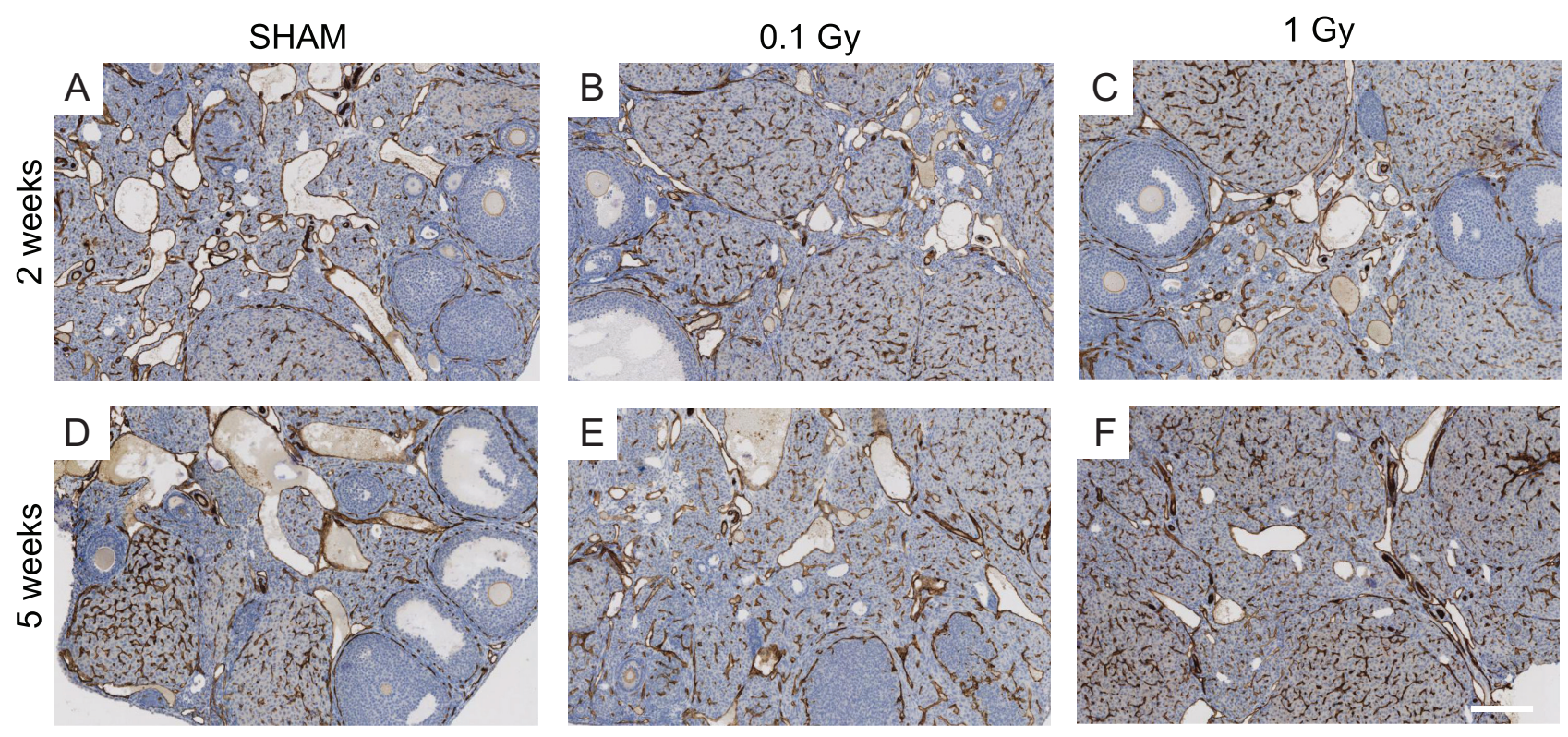

Figure 6 The ovarian vasculature does not undergo prominent architectural changes in response to single doses of 0.1 and 1 Gy TBI at 2 and 5 weeks post exposure. Ovaries from ( $A$ and $D$ ) sham mice or mice exposed to ( $B$ and $E$ ) 0.1 Gy or (C and F) 1 Gy radiation were harvested either two weeks (A, B and C) or five weeks (D, E and F) post exposure and histologic sections were processed for automated immunohistochemistry (IHC) with an antibody against the endothelial cell marker CD31. The validation for the use of this antibody for IHC is shown in Supplementary Fig. 4. Representative images are shown. The scale bar is $200 \mu \mathrm{m}$.

Straub et al. 2015). However, the doses of radiation we examined in this study mimic those that occur as scatter doses in typical therapeutic interventions. Thus, in the setting of fertility preservation, it will be particularly important to evaluate the functional ability of the radiation-exposed stromal compartment to fully support long-term follicle and gamete development.

Follicle loss is a common feature of both iatrogenic reproductive aging due to radiation and physiologic reproductive aging, and advanced reproductive age is also associated with increased stromal fibrosis (Wallace et al. 2003, Broekmans et al. 2009, Briley et al. 2016). In our model of radiation-induced damage, complete follicle loss was observed in the absence of detectable fibrosis. This observation may provide important insights into the temporal events of ovarian changes that drive physiologic reproductive aging. For example, age-associated fibrosis in the ovary may be a consequence of follicular loss rather than the cause of it. Studies with this TBI mouse model are ongoing to distinguish between acute vs chronic late impacts of radiation damage on ovarian function and to determine whether the mechanisms of iatrogenic and physiologic reproductive aging are similar or distinct. Furthermore, it is important to acknowledge that in the TBI model, we cannot readily distinguish whether the observed follicle loss is due to direct damage to the ovary or instead due to indirect systemic or abscopal effects. Using the Small Animal Radiation Research Platform, we compared the effects of targeted radiation vs. TBI on the ovary following a single $1 \mathrm{~Gy}$ dose (Grover et al., unpublished observations). We found that at 2 weeks post exposure, there was no significant difference between the ovarian damage elicited by direct vs indirect effects, suggesting that, at least in the short term, there are negligible compounding systemic effects on the ovary.

\section{Supplementary data}

This is linked to the online version of the paper at https://doi.org/10.1530/REP-18-0089.

\section{Declaration of interest}

The authors declare that there is no conflict of interest that could be perceived as prejudicing the impartiality of the research reported.

\section{Funding}

This work was supported by the Center for Reproductive Health After Disease (P50 HD076188; pilot project to F E D) from the National Centers for Translational Research in Reproduction and Infertility (NCTRI). The KUMC Histology Core was supported by the P30 HD002528 (Kansas IDDRC).

\section{Acknowledgments}

The authors would like to thank Jing Huang (KUMC Histology Core) and Megan Larmore (University of Washington Histology and Imaging Core) for their technical assistance in ovarian tissue sectioning and quantitative microscopy analysis, respectively. 


\section{References}

Adriaens I, Smitz J \& Jacquet P 2009 The current knowledge on radiosensitivity of ovarian follicle development stages. Human Reproduction Update 15 359-377. (https://doi.org/10.1093/humupd/ dmn063)

Baker TG 1978 Effects of ionizing radiations on mammalian oogenesis: a model for chemical effects. Environmental Health Perspectives 24 31-37. (https://doi.org/10.1289/ehp.782431)

Briley SM, Jasti S, McCracken JM, Hornick JE, Fegley B, Pritchard MT \& Duncan FE 2016 Reproductive age-associated fibrosis in the stroma of the mammalian ovary. Reproduction 152 245-260. (https://doi. org/10.1530/REP-16-0129)

Bristol-Gould SK, Kreeger PK, Selkirk CG, Kilen SM, Cook RW, Kipp JL, Shea LD, Mayo KE \& Woodruff TK 2006 Postnatal regulation of germ cells by activin: the establishment of the initial follicle pool. Developmental Biology 298 132-148. (https://doi.org/10.1016/j.ydbio.2006.06.025)

Broekmans FJ, Soules MR \& Fauser BC 2009 Ovarian aging: mechanisms and clinical consequences. Endocrine Reviews 30 465-493. (https://doi. org/10.1210/er.2009-0006)

Critchley HO \& Wallace WH 2005 Impact of cancer treatment on uterine function. Journal of the National Cancer Institute Monographs 64-68. (https://doi.org/10.1093/jncimonographs/lgi022)

Duncan FE, Jasti S, Paulson A, Kelsh JM, Fegley B \& Gerton JL 2017 Age-associated dysregulation of protein metabolism in the mammalian oocyte. Aging Cell 16 1381-1393. (https://doi.org/10.1111/acel.12676)

Grigsby PW, Russell A, Bruner D, Eifel P, Koh WJ, Spanos W, Stetz J, Stitt JA \& Sullivan J 1995 Late injury of cancer therapy on the female reproductive tract. International Journal of Radiation Oncology Biology Physics 31 1281-1299. (https://doi.org/10.1016/0360-3016(94)00426-L)

Hutt KJ 2015 The role of BH3-only proteins in apoptosis within the ovary. Reproduction 149 R81-R89. (https://doi.org/10.1530/REP-14-0422).

Kerr JB, Brogan L, Myers M, Hutt KJ, Mladenovska T, Ricardo S, Hamza K, Scott CL, Strasser A \& Findlay JK 2012a The primordial follicle reserve is not renewed after chemical or gamma-irradiation mediated depletion. Reproduction 143 469-476. (https://doi.org/10.1530/REP-11-0430)

Kerr JB, Hutt KJ, Michalak EM, Cook M, Vandenberg CJ, Liew SH, Bouillet P, Mills A, Scott CL, Findlay JK et al. 2012b DNA damageinduced primordial follicle oocyte apoptosis and loss of fertility require TAp63-mediated induction of Puma and Noxa. Molecular Cell 48 343-352. (https://doi.org/10.1016/j.molcel.2012.08.017)

Meirow D, Biederman H, Anderson RA \& Wallace WH 2010 Toxicity of chemotherapy and radiation on female reproduction. Clinical Obstetrics and Gynecology 53 727-739. (https://doi.org/10.1097/ GRF.0b013e3181f96b54)

Morita Y, Perez GI, Paris F, Miranda SR, Ehleiter D, Haimovitz-Friedman A, Fuks Z, Xie Z, Reed JC, Schuchman EH et al. 2000 Oocyte apoptosis is suppressed by disruption of the acid sphingomyelinase gene or by sphingosine-1-phosphate therapy. Nature Medicine 6 1109-1114. (https://doi.org/10.1038/80442)

Nicholson DW, Ali A, Thornberry NA, Vaillancourt JP, Ding CK, Gallant M, Gareau Y, Griffin PR, Labelle M, Lazebnik YA et al. 1995 Identification and inhibition of the ICE/CED-3 protease necessary for mammalian apoptosis. Nature 376 37-43. (https://doi.org/10.1038/376037a0)

Rinaldi VD, Hsieh K, Munroe R, Bolcun-Filas E \& Schimenti JC 2017 Pharmacological inhibition of the DNA damage checkpoint prevents radiation-induced oocyte death. Genetics 206 1823-1828. (https://doi. org/10.1534/genetics.117.203455)

Sanders JE, Hawley J, Levy W, Gooley T, Buckner CD, Deeg HJ, Doney K, Storb R, Sullivan K, Witherspoon R et al. 1996 Pregnancies following high-dose cyclophosphamide with or without high-dose busulfan or total-body irradiation and bone marrow transplantation. Blood $\mathbf{8 7}$ 3045-3052.

Scholzen T \& Gerdes J 2000 The Ki-67 protein: from the known and the unknown. Journal of Cellular Physiology 182 311-322. (https://doi.org/10.1002/(SICI)1097-4652(200003)182:3<311::AIDJCP1>3.0.CO;2-9)

Straub JM, New J, Hamilton CD, Lominska C, Shnayder Y \& Thomas SM 2015 Radiation-induced fibrosis: mechanisms and implications for therapy. Journal of Cancer Research and Clinical Oncology 141 1985-1994. (https://doi.org/10.1007/s00432-015-1974-6)

Wallace WH, Shalet SM, Crowne EC, Morris-Jones PH \& Gattamaneni HR 1989 Ovarian failure following abdominal irradiation in childhood: natural history and prognosis. Clinical Oncology 1 75-79. (https://doi. org/10.1016/S0936-6555(89)80039-1)

Wallace WH, Thomson AB \& Kelsey TW 2003 The radiosensitivity of the human oocyte. Human Reproduction 18 117-121. (https://doi. org/10.1093/humrep/deg016)

Weintraub NL, Jones WK \& Manka D 2010 Understanding radiationinduced vascular disease. Journal of the American College of Cardiology 55 1237-1239. (https://doi.org/10.1016/j.jacc.2009.11.053)

Wo JY \& Viswanathan AN 2009 Impact of radiotherapy on fertility, pregnancy, and neonatal outcomes in female cancer patients. International Journal of Radiation Oncology Biology Physics 73 1304-1312. (https://doi.org/10.1016/j.ijrobp.2008.12.016)

Yu J, Hecht NB \& Schultz RM 2002 RNA-binding properties and translation repression in vitro by germ cell-specific MSY2 protein. Biology of Reproduction 67 1093-1098. (https://doi.org/10.1095/ biolreprod67.4.1093)

Zelinski MB, Murphy MK, Lawson MS, Jurisicova A, Pau KY, Toscano NP, Jacob DS, Fanton JK, Casper RF, Dertinger SD et al. 2011 In vivo delivery of FTY720 prevents radiation-induced ovarian failure and infertility in adult female nonhuman primates. Fertility and Sterility 95 1440-5.e17-1445.e1-7. (https://doi.org/10.1016/j.fertnstert.2011.01.012)

Received 17 February 2018

First decision 19 March 2018

Revised manuscript received 2 April 2018

Accepted 10 April 2018 